\title{
Data Fusion through Fuzzy-Bayesian Networks for Belief Generation in Cognitive Agents
}

\author{
Fusão de Dados Através de Redes Fuzzy-Bayesianas para a Construção de Crenças de \\ Percepção em Agentes Cognitivos
}

\author{
Munyque Mittelmann ${ }^{1 *}$, Jerusa Marchi ${ }^{1}$, Aldo von Wangenheim ${ }^{1}$
}

\begin{abstract}
Situation Awareness provides a theory for agents decision making to allow perception and comprehension of his environment. However, the transformation of the sensory stimulus in beliefs to favor the $\mathrm{BDI}$ reasoning cycle is still an unexplored subject. Autonomous agent projects often require the use of multiple sensors to capture environmental aspects. The natural variability of the physical world and the imprecision contained in linguistic concepts used by humans mean that sensory data contain different types of uncertainty in their measurements. Thus, to obtain the Situational Awareness for Agents with physical sensors, it is necessary to define a data fusion process to perform uncertainty treatment. This paper presents a model to beliefs generation using fuzzy-bayesian inference. An example in robotics navigation and location is used to illustrate the proposal.
\end{abstract}

Keywords: Situation Awareness — Data Fusion - Belief Generation — Fuzzy-Bayesian Networks

Resumo: A área de Consciência Situacional provê uma teoria que embasa a tomada de decisão em agentes, de forma a permitir a percepção e a compreensão do ambiente em que o agente está inserido. Contudo, a transformação de estímulos sensoriais em crenças que favoreçam o ciclo de raciocínio em agentes BDI ainda é uma área pouco explorada. Projetos de agentes autônomos frequentemente demandam a utilização de múltiplos sensores para possibilitar a captura de aspectos do ambiente. A variabilidade natural do mundo físico e a imprecisão contida em conceitos linguísticos utilizados por humanos fazem com que os dados sensoriais contenham diferentes tipos de incerteza em suas medidas. Deste modo, para possibilitar a obtenção da Consciência Situacional de Agente com sensores físicos, é necessário definir um processo de fusão de dados que realize tratamento de incerteza. Este trabalho apresenta um modelo para geração de crenças, utilizando inferência Fuzzy-Bayesiana. Para ilustrar a proposta, um exemplo em navegação e localização robótica é utilizado.

Palavras-Chave: Consciência Situacional - Fusão de Dados - Geração de Crenças - Redes FuzzyBayesianas

${ }^{1}$ Departamento de Informática e Estatística, Universidade Federal de Santa Catarina (UFSC), Brasil

*Corresponding author: munyquee@gmail.com

DOI: http://dx.doi.org/10.22456/2175-2745.87085 • Received: 18/12/2018 • Accepted: 14/04/2019

CC BY-NC-ND 4.0 - This work is licensed under a Creative Commons Attribution-NonCommercial-NoDerivatives 4.0 International License.

\section{Introdução}

Na área de psicologia cognitiva, a avaliação da situação atual é considerada como um aspecto cognitivo necessário para que haja uma tomada de decisão efetiva [1]. Neste sentido, a Consciência Situacional (Situation Awareness - SA) é definida como a percepção dos elementos no ambiente dentro de um volume espaço-temporal e a compreensão de seus significados e de sua projeção num futuro próximo [2].

Endsley [2] define um modelo de SA que incorpora três diferentes níveis de consciência: (i) a percepção de sinais, (ii) a compreensão e integração da informação e (iii) a projeção da informação em eventos futuros. Embora a abordagem original seja referente a SA humana, o modelo também é utilizado como uma justificativa para estruturar o processo computacional de consciência situacional [3]. A diferença entre o processo de consciência situacional humano e o computacional é que o processo humano precisa ser medido e possivelmente suportado, enquanto que o processo computacional precisa ser definido e implementado [3].

O primeiro nível de SA é a Percepção e envolve perceber os sinais referentes aos atributos e dinâmicas de elementos relevantes no ambiente. O procedimento de coleta e monitoramento de dados situacionais varia dependendo das partic- 
ularidades do cenário de aplicação, podendo incluir redes de sensores, dispositivos e atuadores [4].

O nível intermediário de SA é a Compreensão, que é responsável por integrar e compreender o significado das informações percebidas, sendo composto pela agregação de situações (associação), medição das propriedades das situações (avaliação), estimação de situações (inferência) e medições de capacidade, oportunidade e intenção da situação (avaliação de impacto) [5].

Por fim, o nível de Projeção é responsável por projetar a informação percebida e compreendida em situações futuras. Trabalhos na área de agentes autônomos costumam utilizar a projeção para a tomada de decisão e planejamento do agente [6].

Agentes autônomos usualmente empregam múltiplos sensores para possibilitar a captura de diferentes aspectos do ambiente. Nestes casos, é necessário que o agente tenha a capacidade de realizar um processo conhecido como fusão de dados, que inclui a fusão dos dados de baixo nível produzidos por sensores físicos, o reconhecimento de entidades relacionadas ao contexto para formar uma figura unificada do ambiente e identificação das correlações existentes entre entidades e situações [5, 7].

A pesquisa na área de Fusão de Informação fraciona o processo de fusão de dados sensoriais em quatro níveis funcionais, que são detalhados na Seção $2.1[8,7]$. Os níveis de fusão de dados podem ser relacionados aos níveis de SA propostos por Endsley [2], uma vez que a fusão de dados de baixo nível é inerente ao nível de Percepção do agente e o produto do processo de fusão de dados de alto nível corresponde a ter alcançado a Consciência Situacional do agente [5].

Ao nível de Percepção, os dados capturados por sensores físicos normalmente são afetados por algum nível de imprecisão ou incerteza em suas medidas. Tratar a incerteza proveniente de dados sensoriais é um dos desafios que impulsionam a pesquisa em fusão de dados [9].

A variabilidade natural do mundo físico acarreta na existência de imprecisão por aleatoriedade nos dados sensoriais, que é independente do conhecimento de especialistas no domínio [10]. Outro tipo de incerteza comum ao utilizar sensoriamento é a incerteza por vagueza, que ocorre devido à imprecisão contida nos conceitos linguísticos usados por humanos (e.g. alto, pequeno, distante), que não podem são precisamente definidos por limiares numéricos.

Tendo em vista os diferentes tipos de incerteza contidas em informações sensoriais, diferentes trabalhos na literatura descrevem métodos para permitir a obtenção de Consciência Situacional sobre dados com incerteza. Em [11, 12, 13] são utilizadas Redes Bayesianas para Avaliação de Situação a partir de dados que contenham apenas a incerteza por aleatoriedade. Em contrapartida, os abordagens apresentadas em [14, 15, 16] empregam a Teoria Fuzzy para modelar a obtenção da Consciência Situacional a partir de dados com incerteza por vagueza. Para tratar simultaneamente a incerteza por vagueza e por aleatoriedade, $[17,18,19,20,21,22]$ uti- lizam modelos híbridos Fuzzy-Bayesianos para Avaliação de Situação, permitindo a realização da inferência Bayesiana a partir de dados descritos por Conjuntos Fuzzy. Estes trabalhos realizam inferência dicotômica, que considera que uma observação possa pertencer apenas a até dois conjuntos Fuzzy (e.g. Baixo e Médio). Entretanto, em um cenário real, existem fenômenos que não podem ser modelados por termos booleanos. Por exemplo, no contexto de análise de peso em pacientes apresentado em [23], a magreza é classificada por meio dos conjuntos Fuzzy "extrema", "grave" e "elevada", que possuem uma sobreposição semântica. Neste caso, a situação de um paciente poderia ter um grau de pertinência aos três conjuntos Fuzzy supracitados. As abordagens de obtenção de Consciência Situacional apresentadas nos trabalhos relacionados não fornecem fundamentos para tratar a inferência não-dicotômica, assim, carecem na modelagem de situações como a descrita no exemplo.

Desta forma, este trabalho tem o objetivo de prover o nível de Percepção na Consciência Situacional de um agente, integrando a inferência Fuzzy-Bayesiana não-dicotômica de Brignoli [23, 24] a um modelo de Fusão de Dados. Neste modelo, as percepções do agente são representadas por um conjunto de crenças, gerado como saída do modelo. As crenças de Percepção possuem grau de certeza e são propagadas para os níveis de Compreensão e Projeção.

O trabalho está estruturado como segue: na Seção 2 são apresentados os conceitos referentes aos níveis Funcionais de Fusão de Dados e a descrição da inferência Fuzzy-Bayesiana; na Seção 3 é apresentada a proposta de modelo paralelamente a um cenário exemplificando sua aplicação; a Seção 4 apresenta a implementação do exemplo em Jason; a Seção 5 descreve a modelagem e simulações da Inferência FuzzyBayesiana; por fim, na Seção 6 são apresentadas algumas considerações da pesquisa.

\section{Conceitos Básicos}

Apresenta-se nesta seção a distinção entre os níveis de fusão de dados e sua relação com os níveis de SA encontrados nos trabalhos de Endsley [2], e posteriormente, descreve-se a inferência Fuzzy-Bayesiana que foi empregada nesta pesquisa dentro de um modelo de fusão de dados.

\subsection{Níveis Funcionais de Fusão de Dados}

A classificação mais comum do processo de fusão de dados é o modelo JDL proposto por White [8] que considera o processo de fusão em quatro níveis crescentes de abstração: Objeto, Situação, Impacto e Refinamento do Processo.

Como extensão do modelo JDL, Steinberg e Bowman [7] apresentam os níveis Funcionais de Fusão de Dados (FFD). Neste particionamento, uma entidade de interesse do sistema pode ser vista como (i) um indivíduo com atributos, características e comportamentos ou (ii) como um conjunto de componentes inter-relacionados. Os níveis FFD de Steinberg e Bowman [7] são definidos conforme segue: 
- (0-FFD) Avaliação de Sinais: estimação e predição de sinais ou estados característicos;

- (1-FFD) Avaliação de Entidades: estimação e predição de parametrização de entidade e estados atributivos;

- (2-FFD) Avaliação de Situações: estimação e predição das estruturas de partes da realidade, como relação entre entidades e suas implicações nos estados de entidades relacionadas;

- (3-FFD) Avaliação de Impacto: estimação e predição do custo/benefício do sinal, entidade ou estados de situações;

- (4-FFD) Avaliação de Desempenho: estimação e predição do desempenho do sistema quando comparado aos estados desejados e medidas de efetividade.

A fusão de dados de baixo nível corresponde aos níveis 0FFD, 1-FFD e 2-FFD, os quais são responsáveis pela identificação de entidades e suas correlações. Estes três níveis são análogos ao nível de Percepção de SA. O nível 2-FFD também inclui a estimação da implicação dos relacionamentos entre entidades em seus estados, podendo ser relacionado ao nível de Compreensão de SA. Os níveis 3-FFD e 4-FFD têm a função de prever custos, estados e desempenho do sistema, tendo correspondência, principalmente, com o nível de Projeção de SA.

Relacionamentos entre entidades no nível 2-FFD podem ter características implicacionais e são comumente modelados por meio de Modelos Gráficos Probabilísticos, como Redes Bayesianas, Redes Bayesianas Dinâmicas e Redes FuzzyBayesianas $[25,5]$. Com intuito de compreender a fusão de dados de baixo nível e o nível de Percepção de SA, este trabalho aplica inferência Fuzzy-Bayesiana em um modelo para agentes.

\subsection{Inferência Fuzzy-Bayesiana}

Conjuntos difusos são classes que possuem continuidade em seus graus de pertinência [26]. Dados difusos usualmente não são descritos pela inferência Bayesiana padrão e, assim, é necessário modelá-los de modo a incorporar seu aspecto difuso antes de analisá-los por métodos estatísticos [27, 28]. Os estudos de Viertl [27, 29, 30, 31, 28], investigam a generalização da estatística Bayesiana para dados difusos, tais abordagens híbridas são conhecidas como inferências Fuzzy-Bayesianas.

Dentre as inferências Fuzzy-Bayesianas existentes, o modelo apresentado por Brignoli et al. [24] é uma extensão que visa incluir variáveis difusas não-dicotômicas ${ }^{1}$. O modelo de inferência Bayesiana com entradas difusas proposto é baseado na superposição de estados. Brignoli et al. [24] define a probabilidade $\widetilde{P}$ sobre evidências imprecisas, conforme a seguinte equação:

\footnotetext{
${ }^{1}$ Considera-se como variável dicotômica aquela que é dividida logicamente em apenas dois conceitos, geralmente opostos.
}

$$
\begin{aligned}
& \widetilde{P}\left(H_{i} \mid E_{j} x_{k}\right)= \\
& \frac{P\left(H_{i}\right) \times \prod_{j=1}^{m} \sum_{k=1}^{t}\left(P\left(E_{j} x_{k} \mid H_{i}\right) \times\left(\varepsilon E_{j} x_{k}\right)\right)}{\sum_{l=1}^{n} P\left(H_{l}\right) \times \prod_{j=1}^{m} \sum_{k=1}^{t}\left(P\left(E_{j} x_{k} \mid H_{l}\right) \times\left(\varepsilon E_{j} x_{k}\right)\right)}
\end{aligned}
$$

Na qual, $H_{i}$, com $1 \leq i \leq n$, é o vetor de $n$ hipóteses; $E_{j} x_{k}$, com $1 \leq j \leq m$ e $1 \leq k \leq t$, é o vetor de $m$ evidências $E$ observadas nos estados $x$ contínuos; $t$ é a quantidade de estados; $P\left(H_{i}\right)$ é a probabilidade a priori sobre $H_{i} ; P\left(E_{j} x_{k} \mid H_{i}\right)$ é a probabilidade condicional de $E_{j} x_{k}$ dado $H_{i}$; e $\varepsilon E_{j} x_{k}$ representa o estado $x_{k}$ da evidência $E_{j}$ em relação a função de pertinência difusa. Assim, $\widetilde{P}\left(H_{i} \mid E_{j} x_{k}\right)$ é o vetor das probabilidades de $H_{i}$ ajustadas pela imprecisão sobre as variáveis $E_{j}$ de estados contínuos $x_{k}$.

O modelo de inferência Fuzzy-Bayesiana de [24] é empregado nesta proposta. A inferência Fuzzy-Bayesiana é utilizada para tratar a incerteza por vagueza presente nas entidades observadas e para modelar os relacionamentos probabilísticos entre entidades.

\section{Proposta}

O trabalho propõe um modelo de Fusão de Dados com Incerteza para atingir a Consciência Situacional de um agente ao nível de Percepção. O modelo abrange os níveis de fusão de dados 0-FFD, 1-FFD e 2-FFD. Para esclarecer a proposta, é introduzido um cenário de exemplo na Seção 3.1. O cenário de exemplo é acompanhado em paralelo ao detalhamento da proposta. A Figura 1 introduz uma visão geral do modelo de Fusão de Dados, ilustrando sua relação com os modelos de SA e FFD.

No nível 0-FFD, é realizada a captura das medições sensoriais, são definidas as estruturas dos conjuntos de medição, mas não são tratadas suas semânticas. O nível 1-FFD é responsável pela estimação de entidades e classificações. As entidades são agregadas e consideradas como observações, em seguida, são classificadas em relação a sua pertinência a conjuntos fuzzy do domínio.

O nível 2-FFD diz respeito as relações entre entidades e suas implicações. A implicação entre as entidades é realizada por meio da inferência Bayesiana com as entradas difusas do nível predecessor. Com base nos resultados da inferência, o modelo gera crenças, com grau de certeza, sobre a situação observada. Neste modelo, os níveis 0-FFD, 1-FFD e 2-FFD representam o nível de Percepção de SA. As crenças geradas pelo modelo são disponibilizadas para serem utilizadas nos níveis de Compreensão e Projeção de SA. Os estágios do modelo são especificadas nas Seções 3.2, 3.3 e 3.4.

\subsection{Exemplo de Cenário de Aplicação}

Considere a existência de um robô cujo objetivo é limpar os cômodos de uma casa. Como cada cômodo demanda equipamentos de limpeza distintos, o robô precisa saber em qual cômodo está. A casa é composta por dois quartos $\left(Q_{1}\right.$ e $Q_{2}$, 


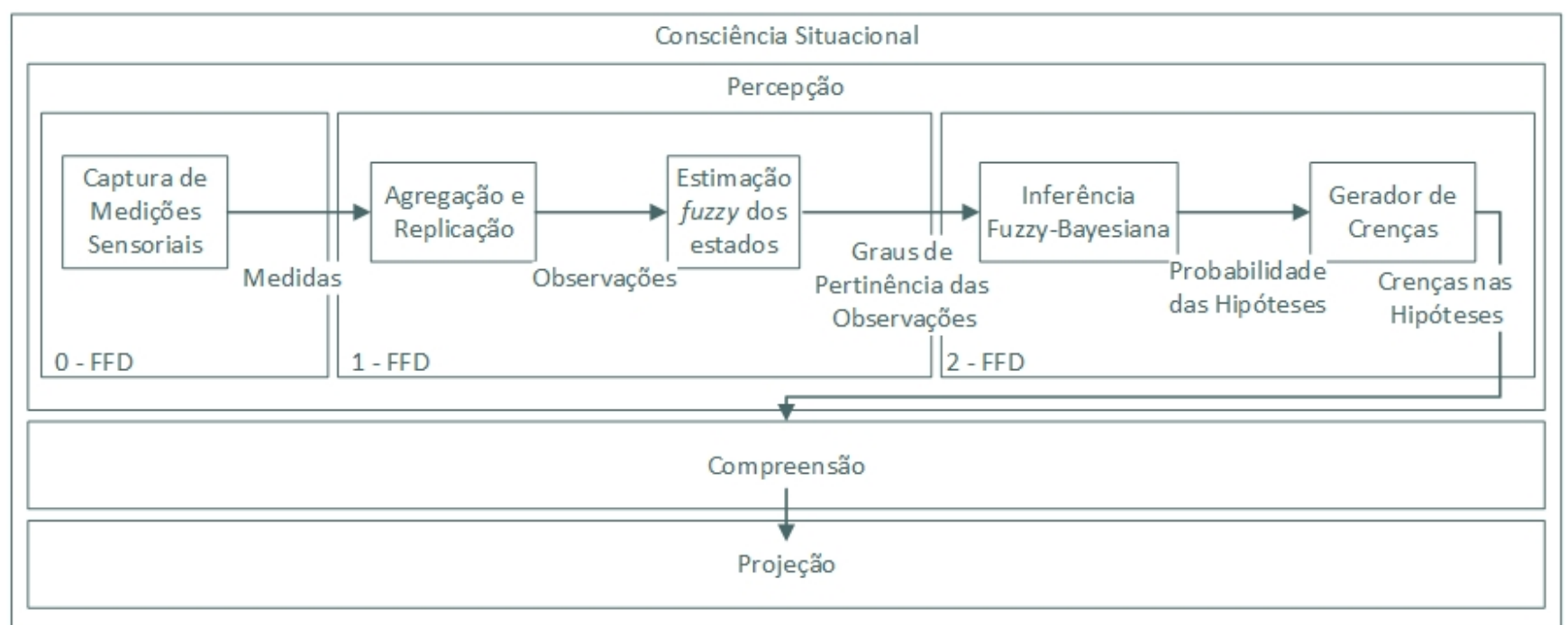

Figura 1. Modelo de Fusão de Dados para Consciência Situacional

respectivamente) e dois corredores conectando os quartos $\left(C_{1}\right.$ e $C_{2}$, respectivamente). A Figura 2(a), ilustra a distribuição dos cômodos $Q_{1}, Q_{2}, C_{1}$ e $C_{2}$ pela casa.

$\mathrm{O}$ robô caminha aleatoriamente pela casa e não conhece a disposição de seus cômodos. Para perceber o ambiente, o robô possui apenas sensores para captar sons, luminosidade e distância. Com estes sensores, o robô precisa descobrir, respectivamente, se a sonorização do ambiente é fraca, média ou elevada (Figura 2(b)), se o ambiente está claro ou escuro (Figura 2(c)) e se o espaço entre as paredes é amplo ou apertado (Figura 2(d)). Observa-se que as informações sensoriais são classificadas por variáveis linguísticas providas de imprecisão por vagueza (fraca, média e elevada para a variável intensidade sonora, claro, médio e escuro para a iluminação, amplo e apertado para o espaço).

Antes de ser posto em funcionamento, o robô recebeu informações amostrais sobre mapeamentos anteriores do ambiente. Assim, ele conhece a probabilidade a priori de estar em cada cômodo, bem como as probabilidades condicionais de estar com sonorização fraca, média ou elevada, em um ambiente com iluminação clara, média ou escura e em um espaço amplo ou apertado dado que está em algum cômodo específico. As Figuras 2(b), 2(c) e 2(d) ilustram, respectivamente, a distribuição da sonorização, iluminação e espaço pela casa em um determinado momento. Os valores das medições de som, luminosidade e distância podem variar dependendo do momento, seja por causa de falha sensorial ou de mudança no ambiente.

Munido apenas das informações sensoriais e das probabilidades a priori e condicionais, o robô deve decidir em qual cômodo está e, assim, ser capaz de determinar seus planos de ação. Durante a apresentação da proposta, o cenário do Robô Limpador é retomado de modo a exemplificar a aplicação e os níveis do modelo. A proposta é apresentada em três estágios, correspondentes aos níveis FFD abrangidos pelo modelo.

\subsection{Nível de Avaliação de Sinais (0-FFD)}

No nível 0-FFD, é realizada a estimação de sinais ou medições. O Agente possui um conjunto $\mathscr{S}_{T}=\left\{S_{T_{1}}, \ldots, S_{T_{n}}\right\}$ de sensores, onde $T_{i}$ representa o tipo de sensor e $S_{T_{i}}=\left\{s_{1}, \ldots, s_{m}\right\}$ representa um conjunto de sensores físicos de mesmo tipo. Para cada $S_{T_{i}}$ são obtidos conjuntos $M_{i}$ de medidas, de forma que $\mathscr{M}=\bigcup_{i \in T} M_{i}$ forma o conjunto de todos os sinais percebidos pelo agente.

Exemplo 1 O Robô Limpador da Seção 3.1 possui três tipos de sensores: luminosidade, distância e sonoro, ou seja: $\mathscr{S}_{T_{\text {robo }}}$ $=\left\{S_{\text {luz }}, S_{\text {distancia }}, S_{\text {som }}\right\}$. O robô possui um sensor de luminosidade, dois de distância e um sonoro, assim $S_{l u z}=\left\{s_{l u z}\right\}$, $S_{\text {distancia }}=\left\{s_{d 1}, s_{d_{2}}\right\}$, com $s_{d 1}$ e $s_{d_{2}}$ sendo, respectivamente, os sensores de distância à direita e à esquerda do robô $\hat{o}^{2}$, e $S_{\text {som }}=\left\{s_{\text {som }}\right\}$. As medidas obtidas pelos sensores são $\mathscr{M}_{\text {robo }}=\left\{m_{\text {luz }}, m_{d 1}, m_{d 2}, m_{\text {som }}\right\}$. A Figura 3 ilustra o nível de Avaliação de Sinais para o Robô Limpador.

\subsection{Nível de Avaliação de Entidades (1-FFD)}

O nível 1-FFD é responsável pela estimação de evidências e suas classificações. Neste estágio, ocorre a transformação dos dados de medidas em evidências observadas. A transformação das medidas é definida como a aplicação de uma função $f(x) \longrightarrow \mathbb{R}$ sobre os mesmos, onde $f \in\{A, R\}$ é uma função de Agregação ou Replicação, $x \in 2^{\mathscr{A} \backslash \emptyset}$ é a aridade da função. Cada função $f(x)$ gera uma nova evidência observada $o_{k} \in \mathbb{R}$, na qual $k$ representa um domínio (ex.: distância, iluminação, sonorização).

A função de Agregação $A$ é realizada por meio de operações sobre uma ou mais medições pertencentes a $\mathscr{M}$ e é definida por $A\left(m_{i}, \ldots, m_{j}\right) \longrightarrow \mathbb{R}$, com $\left(m_{i}, \ldots, m_{j}\right) \in 2^{\mathscr{M} \backslash \emptyset}$. A função de Replicação $R$ realiza cópia de um elemento de $\mathscr{M}$, tal que $R\left(m_{i}\right) \longrightarrow \mathbb{R}, \operatorname{com} m_{i} \in \mathscr{M}$.

\footnotetext{
${ }^{2}$ Optou-se pela utilização de apenas dois sensores de distância para manter o exemplo simples e ilustrativo.

${ }_{3} 2^{\mathscr{M} \backslash \emptyset}$ representa o conjunto das partes de $\mathscr{M}$ sem o conjunto vazio $\emptyset$.
} 


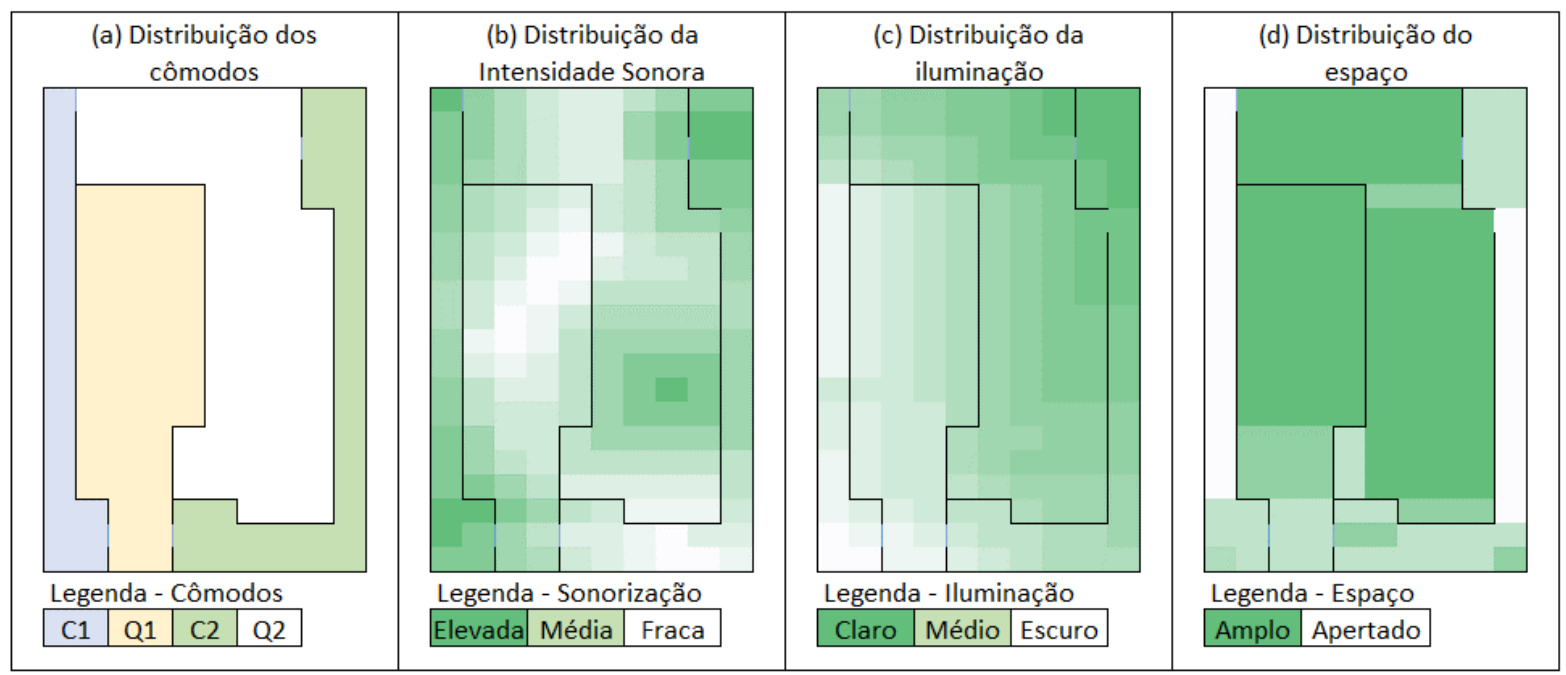

Figura 2. Cenário do Robô Limpador

Sobre $o_{k}$ é aplicado o operador de normalização, tal que $N: \mathbb{R} \longrightarrow[0,1]$. Tal operação visa facilitar o suporte de cada evidência observada, entretanto, não é necessário realizar a normalização. Para fins de simplificação da notação, continuaremos a denominar a evidência observada normalizada de $o_{k}$. Consideraremos $\mathscr{O}=\bigcup_{\forall k} o_{k}$ o conjunto global de evidências.

Exemplo 2 No cenário do Robô Limpador, as medições $m_{d 1}$ e $m_{d 2}$ são agregadas tal que $o_{\text {espaco }}=\left\|m_{d 1}\right\|+\left\|m_{d 2}\right\|^{4}$. Ou seja, o espaço entre as paredes o espaco é a soma da distância da parede à direita do robô com a distância da parede à esquerda do robô. As medições $m_{l u z}$ e $m_{\text {som }}$ são replicadas como evidências observadas, tal que $o_{l u z}=m_{l u z}$ e $o_{\text {som }}=$ $m_{\text {som. }}$. Nota-se que $\mathscr{O}_{\text {robo }}=\left\{o_{\text {luz }}, o_{\text {espaco }}, o_{\text {som }}\right\}$ é o conjunto normalizado de todas as evidências observadas pelo robô.

Para cada evidência observada $o_{k}$ é considerado um universo de discurso no intervalo $\left[\min _{k}, \max _{k}\right]$, denotado por $\mathscr{A}_{k}$, onde $\min _{k}$ e $\max _{k}$, respectivamente, são os valores mínimos e máximos do domínio K. Para cada $o_{k} \in \mathscr{O}$ será calculado o valor de pertinência a algum conjunto fuzzy $A_{k}$, definido sobre $\mathscr{A}_{k}=\left\{A_{k_{1}}, \ldots, A_{k_{n}}\right\}$. O grau de pertinência de $o_{k}$ ao conjunto $A_{k_{i}}$ é denotado por $\mu_{A_{k_{i}}}\left(o_{k}\right)$. O conjunto de todos os graus de pertinência $\mu_{A_{k_{i}}}\left(o_{k}\right), \forall A_{k_{i}} \in \mathscr{A}_{k}$ e $\forall o_{k} \in \mathscr{O}$ é denotado por: $\mu(\mathscr{O})=\bigcup_{\forall k, \forall i} \mu_{A_{k_{i}}}\left(o_{k}\right)$.

Exemplo 3 Para cada evidência observada pelo Robô Limpador, foi definido um universo de discurso e calculados os graus de pertinência da evidência a cada conjunto do universo de discurso. Para o $o_{\text {luz }}, \mathscr{A}_{\text {luz }}=\{$ claro;médio;escuro $\}$. Para $o_{\text {espaco }}, \mathscr{A}_{\text {espaco }}=\{$ amplo $;$ apertado $\}$. Para $o_{\text {som }}, \mathscr{A}_{\text {som }}=$ $\left\{\right.$ baixo; médio; alto\}. A seguir, para cada evidência $o_{k}$ observada pelo robô, foi estimado seu grau de pertinência $\mu_{A_{k_{i}}}\left(o_{k}\right)$ para cada conjunto $A_{k_{i}} \in \mathscr{A}_{k}$ (Figura 4). Como exemplifica a Figura 4, os conjuntos fuzzy $A_{k_{i}}$ podem ser não-dicotômicos,

\footnotetext{
${ }^{4}\|x\|$ representa o módulo de um número $x \in \mathbb{R}$.
}

com sobreposição de estados, o que ocorre nos conjuntos fuzzy nos universos de discurso das observações $o_{l u z}$ e $o_{\text {som. }}$.

\subsection{Nível de Avaliação de Situação (2-FFD)}

O nível 2-FFD é responsável por estimar as relações entre as evidências e suas implicações. Os graus de pertinência das observações $\forall \mu_{A_{k_{i}}}\left(o_{k}\right) \in \mu(\mathscr{O})$, são considerados como as evidências da inferência Fuzzy-Bayesiana . Sendo $\mathscr{H}=$ $\left\{H_{1}, \ldots, H_{n}\right\}$ o conjunto de hipóteses sobre $Z, Z$ como a situação na qual o agente está, $P\left(H_{h}\right) \longrightarrow[0 ; 1]$ representa a probabilidade a priori da $h$-ésima hipótese e $P\left(o_{k} \mid H_{h}\right) \longrightarrow[0 ; 1]$ é a probabilidade de observar $o_{k}$ dada a hipótese $H_{h}$. A probabilidade da hipótese $H_{h}$ ajustada pela imprecisão sobre todas as observações $o_{k} \in \mathscr{O}$ é denotada por $\widetilde{P}\left(H_{h} \mid \mathscr{O}\right)$ e definida aplicando a Equação (1) às definições do modelo, tal que:

$$
\widetilde{P}\left(H_{h} \mid \mathscr{O}\right)=\frac{P\left(H_{h}\right) \times \prod_{k=1}^{|\mathscr{O}|} \sum_{i=1}^{n}\left(P\left(o_{k} \mid H_{h}\right) \times \mu_{A_{k_{i}}}\left(o_{k}\right)\right)}{\sum_{l=1}^{|H|} P\left(H_{l}\right) \times \prod_{k=1}^{|\mathscr{O}|} \sum_{i=1}^{n}\left(P\left(o_{k} \mid H_{l}\right) \times \mu_{A_{k_{i}}}\left(o_{k}\right)\right)}
$$

A probabilidade ajustada pela imprecisão $\widetilde{P}\left(H_{h} \mid \mathscr{O}\right)$ é estimada $\forall H_{h} \in \mathscr{H}$.

A situação avaliada no cenário da Seção 3.1 é a localização atual do robô, ou seja $Z_{\text {robo }}=$ Local. As hipóteses são as possibilidades de localização, os cômodos da casa, então $\mathscr{H}_{\text {robo }}=\left\{H_{Q_{1}} ; H_{Q_{2}} ; H_{C_{1}} ; H_{C_{2}}\right\}$. Assim, $P\left(H_{h}\right)$ representa as probabilidades a priori do robô estar em cada cômodo da casa. $P\left(o_{k} \mid H_{h}\right)$ são as probabilidades condicionais dos estados de uma observação, ou seja $o_{k}=l u z$, som ou espaco, sabendo-se que está em determinado cômodo $\left(H_{h}\right)$.

Exemplo 4 Para o Robô Limpador, a inferência Fuzzy-Bayesiana descobre as probabilidades $\widetilde{P}\left(H_{h} \mid \mathscr{O}\right)$ de estar em cada

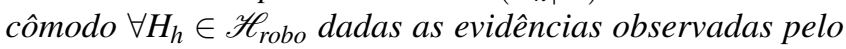
robô $\mathscr{O}_{\text {robo }}=\left\{o_{\text {luz }}, o_{\text {espaco }}, o_{\text {som }}\right\}$. 


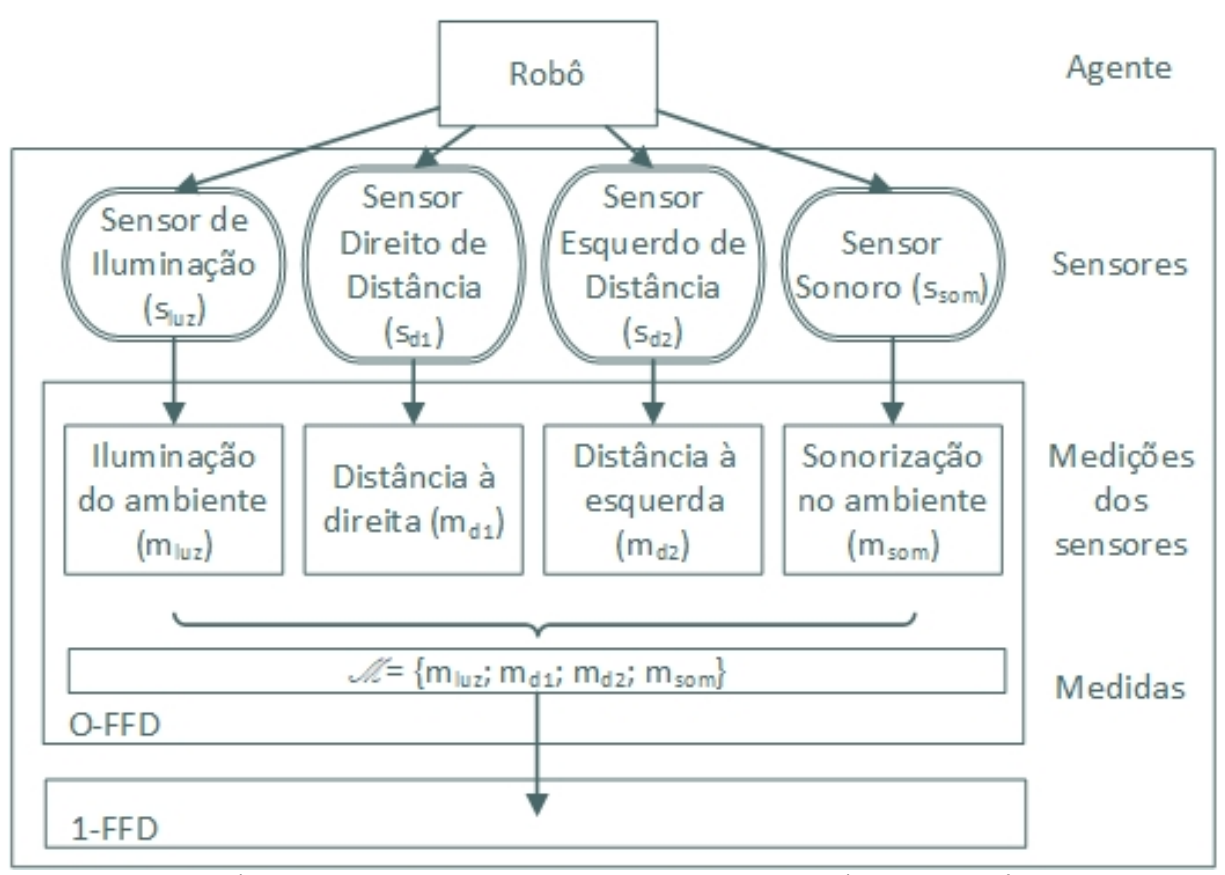

Figura 3. Nível de Avaliação de Sinais (0-FFD) no Cenário do Robô Limpador

A última etapa do modelo é denominada Gerador de Crenças (GC). Considera-se que o conjunto de hipóteses $\mathscr{H}$ seja formado pelas implicações das relações entre as observações, ou seja, a conclusão do que o agente está observando. Assim, para cada hipótese $H_{h} \in \mathscr{H}$, o GC gera uma crença em lógica de predicados na forma: $Z\left(H_{h}, G_{h}\right)$, onde, $Z$ é o símbolo de predicado que representa a situação atual acreditada pelo agente e $G_{h}$ é o grau de certeza do agente em relação a hipótese $H_{h}$, dado por $G_{h}=\widetilde{P}\left(H_{h} \mid \mathscr{O}\right)$.

Exemplo 5 Para cada cômodo da casa, o GC do Robô Limpador gera uma crença com o grau de certeza que o robô tem de estar no cômodo. A Figura 5 ilustra o nível de avaliação de situação no cenário do Robô Limpador.

As crenças geradas pelo modelo são do nível de Percepção e possuem o propósito de fundamentar a integração de crenças distintas como crenças inferidas (nível de Compreensão) e sua projeção em crenças futuras (nível de Projeção). A obtenção de crenças situacionais aos níveis de Percepção, Compreensão e Projeção permite que o agente realize planejamento e tomada de decisão com base em sua situação atual. É intuitivo que o Agente deva considerar, durante a Compreensão e Projeção, a crença com maior grau de certeza. Entretanto, incluir as crenças para todas as hipóteses permite que o agente reaja a situações para as quais nenhuma crença tenha um grau de certeza razoável.

Exemplo 6 Considere um grau de certeza na crença $C_{1}$ de $51 \%$ e em $C_{2}$ de $49 \%$. Estes valores indicam que o agente tem preferência em acreditar em $C_{1}$. Entretanto, como a diferença do grau de certeza entre as crenças é baixa, existe grande incerteza em relação a situação. A propagação desta incerteza permite considerá-la nos planos do agente. Suponha que o agente deva realizar a ação $A_{1}$ quando está na situação descrita por $H_{1}$. Ao invés de executar $A_{1}$ imediatamente, $o$ agente pode reagir de acordo com a incerteza: esperar uma nova informação sensorial, consultar outro agente, entregar o controle da ação a um humano, etc.

\section{Implementação do Agente Robô}

O exemplo explorado ao longo deste trabalho foi implementado em Jason ${ }^{5}$ como um agente Belief-Desire-Intention (BDI). Conforme apresentado na Seção 3.1, o objetivo do robô é limpar os cômodos de uma casa. Para atingir seu objetivo, é necessário que o robô determine em qual cômodo se encontra e escolha os equipamentos adequados para limpeza naquele cômodo. A Figura 6 apresenta uma visão geral da implementação do Robô Limpador, utilizando a notação Prometheus [32].

Conforme ilustrado na Figura 6, a percepção do Robô é representada por crenças no formato local (Comodo, Grau), que representam que o robô acredita que está no local Comodo, com Grau de certeza. Estas crenças representam a saída do Gerador de Crenças definido na Seção 3.4. As ações que o agente pode realizar sobre o ambiente são listadas a seguir:

1. limpar (Agente): o Agente verifica se sua posição atual está suja, neste caso, realiza a limpeza;

2. andar (Agente, Passos): o Agente se locomove Passos posições;

\footnotetext{
${ }^{5}$ Jason é um interpretador para uma versão estendida da linguagem AgentSpeak, disponível em http://jason.sourceforge.net/.
} 


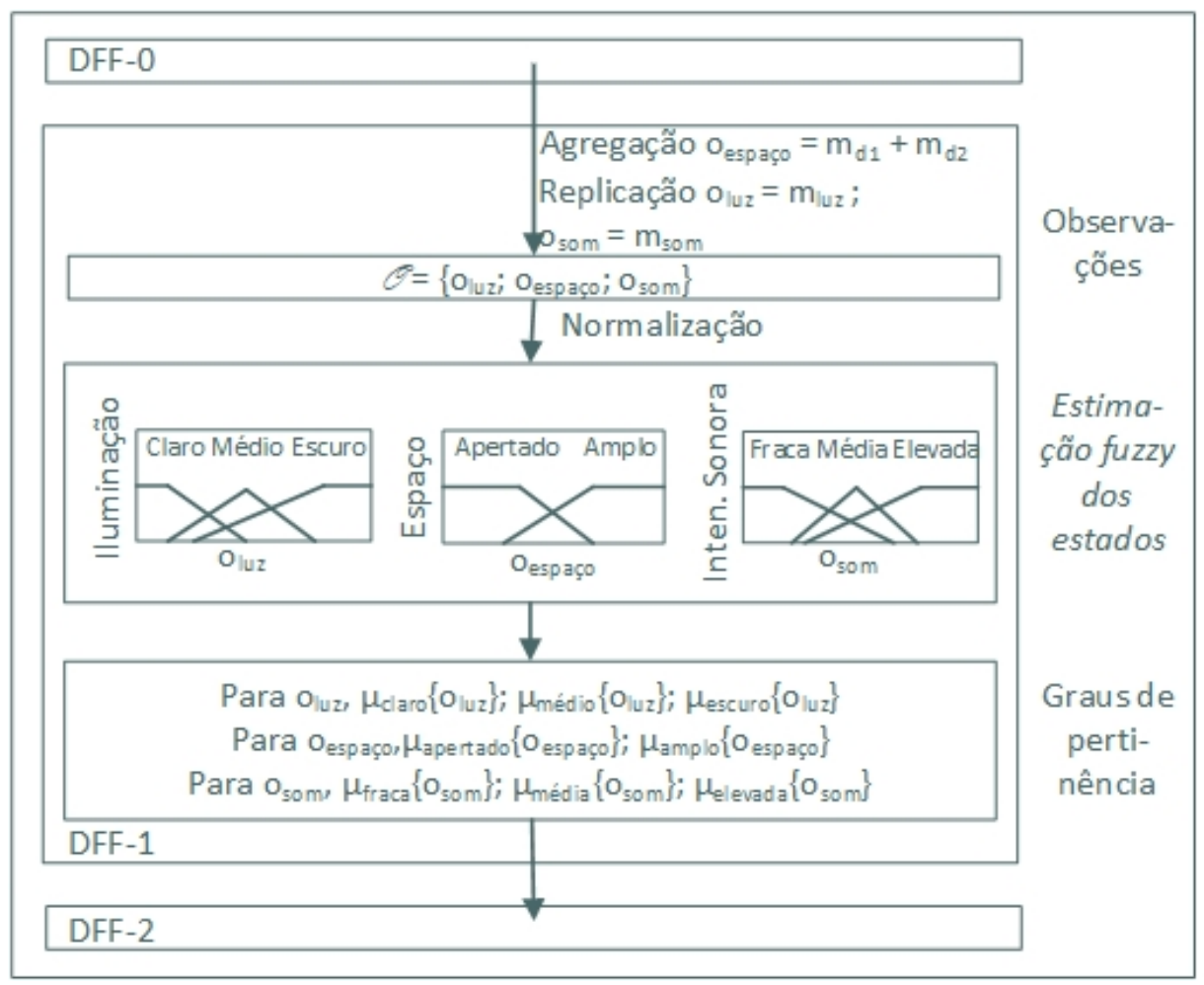

Figura 4. Nível de Avaliação de Entidades (1-FFD) no Cenário do Robô Limpador

3. esperar (Agente, Tempo): o Agente não realiza nenhuma ação durante Tempo segundos;

4. atualizarCrencas (Agente): o Agente atualiza suas crenças, por meio do Modelo de Fusão de Dados proposto na Seção 3, que engloba o processo de obter as medições do ambiente, estimá-las como evidências fuzzy e gerar um conjunto de crenças de percepção no formato local (Comodo, Grau) ) ${ }^{6}$;

5. selecionarMateriais (Agente, Comodo): o Agente seleciona os materiais adequados para realizar limpeza no Comodo.

A base de crenças e planos do agente é composta por uma regra, uma meta inicial e quatro planos de ação, que são apresentados na Figura 7 e detalhados a seguir. A Regra 1 (Figura 7 - linha 2) é verdadeira caso o robô acredite estar em determinado local com grau de certeza maior ou igual a 0,6. Sua meta inicial é ! limpar Ambiente (robo), que representa o objetivo do robô de limpar o ambiente. Para atingir esta meta, o Robô executa o Plano 1 (linhas 8 a 11), que ativa a intenção de ! verificarLocal (robo) e executa as ações limpar (robo) e andar (robo, 1). A intenção ! verificarLocal (robo) ativa o Plano 2 (linhas 14 a 16), que remove as crenças de localização anteriores e atualiza as crenças de acordo com o modelo proposto.

Quando o robô possui a crença local (Comodo, Grau) com grau de certeza de pelo menos 0,6, o Plano 3 (linhas 19

\footnotetext{
${ }^{6}$ Nota-se que o valor de Grau na crença local (Comodo, Grau) é obtido por meio da Equação (2).
}

e 20) é executado. O Plano 3 executa a ação selecionar Materiais (Comodo). Quando o robô não possui nenhuma crença com grau de confiança maior que 0,6 , é executado o Plano 4 (linhas 23 a 25), que realiza a ação para aguardar 5 segundos (esperar (5)) e ativa a intenção de verificar sua localização novamente.

Exemplo 7 No início de uma execução, o modelo de fusão de dados proposto fornece ao robô as seguintes crenças de percepção: local ( 1 , 0.3); local (C2, 0.2); local (Q1, 0.2); local (Q2, 0.3). Como nenhuma das crenças possui grau de certeza igual ou superior a 0,6, a ação do robô é aguardar 5 segundos e atualizar suas crenças, ou seja, o robô segue o Plano 4. Após atualizar suas crenças, o modelo fornece as seguintes percepções ao robô: local (C1, 0.1); local (C2, 0.2) ; local (Q1, $0.6) ; \quad l o c a l(02,0.1)$. Assim, por acreditar estar em $Q_{1}$ com 0.6 de certeza, o robô seleciona os materiais para a limpeza de $Q_{1}$, realiza a limpeza e locomove-se para outra posição, conforme definido pelo Plano 1. O Código 1 lista as ações executadas pelo robô neste exemplo de execução.

\begin{tabular}{|ll|}
\hline [robo] doing: & esperar(robo,5) \\
[robo] doing: & atualizarcrencas (robo) \\
[robo] doing: & selecionarMateriais \\
& (robo, q1) \\
[robo] doing: & limpar (robo) \\
[robo] doing: & andar (robo, 1)
\end{tabular}

Codigo 1. Execução do Robô Limpador como Agente BDI 


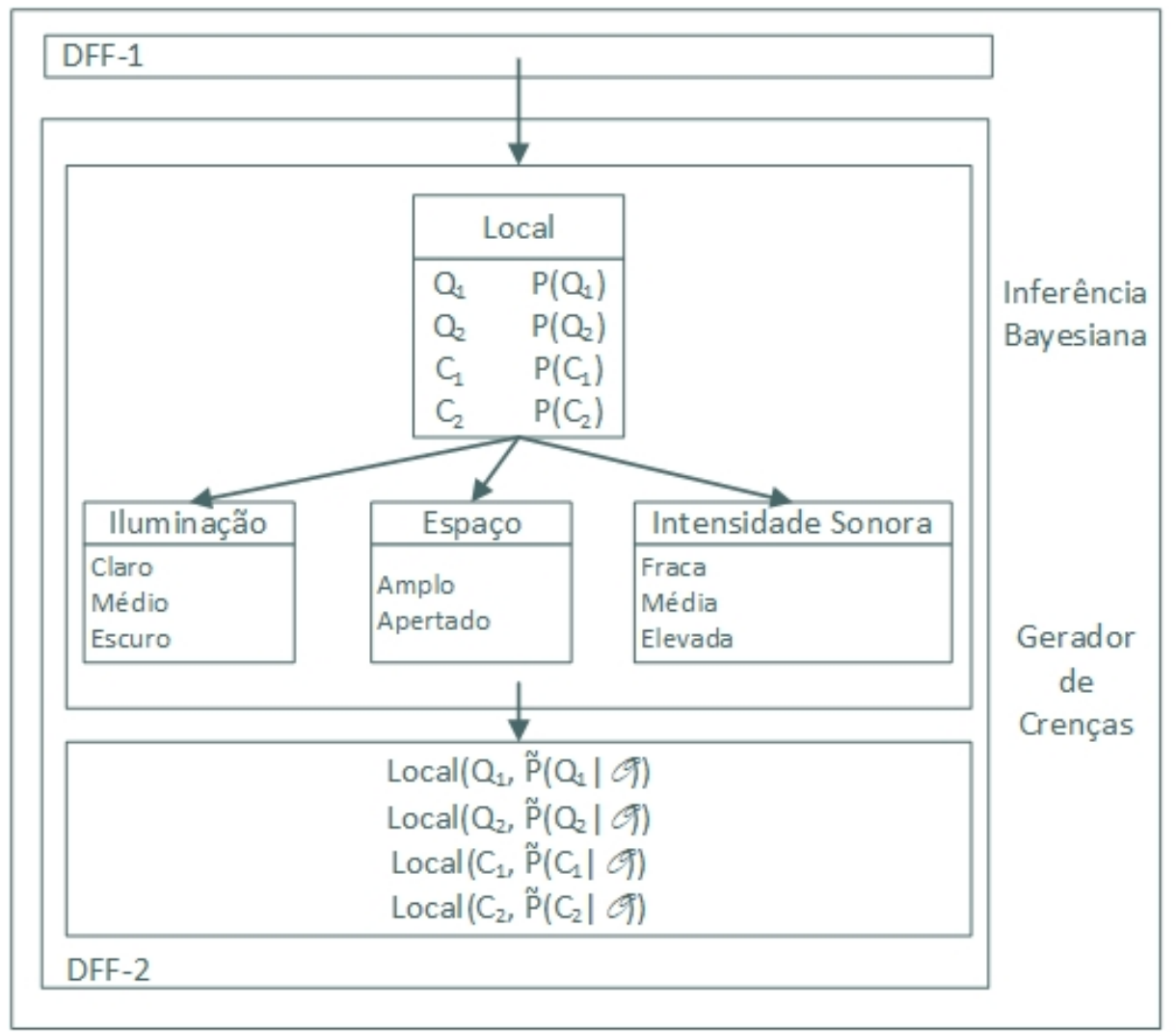

Figura 5. Nível de Avaliação de Situação (2-FFD) no Cenário do Robô Limpador

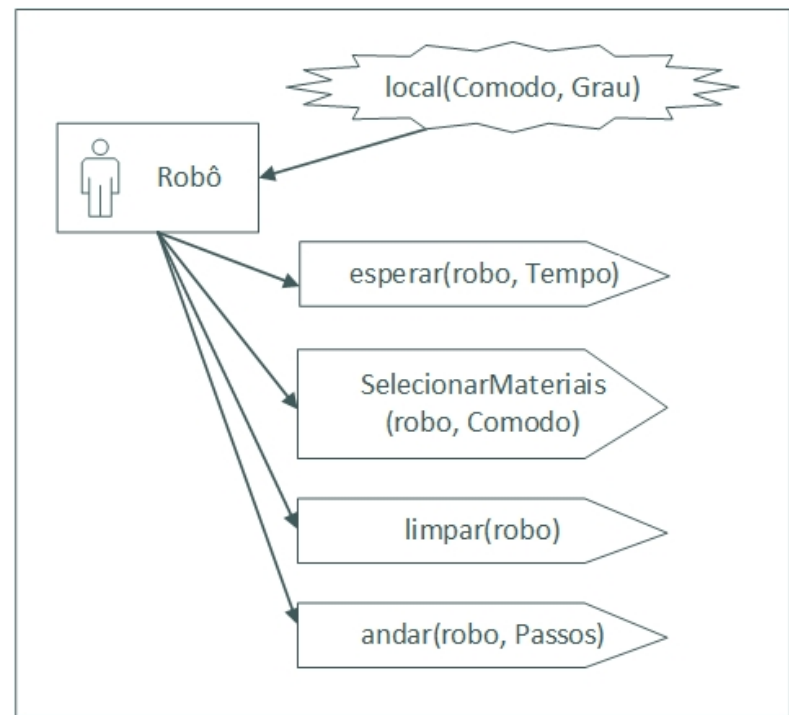

Figura 6. Visão Geral da Implementação Robô Limpador

\section{Modelagem e Simulações da Inferência Fuzzy-Bayesiana}

Esta Seção descreve a modelagem e simulações do cenário do Robô Limpador com a Inferência Fuzzy-Bayesiana. O cenário de exemplo foi modelado por meio de uma Rede Bayesiana de estrutura Naïve Bayes com três variáveis de en-
Tabela 1. Probabilidades Condicionais das Evidências dada a Hipótese

\begin{tabular}{|c|l|l|l|l|l|}
\cline { 3 - 6 } \multicolumn{2}{c|}{} & \multicolumn{5}{c|}{ Local } \\
\cline { 3 - 6 } \multicolumn{2}{c|}{} & Q1 & Q2 & C1 & C2 \\
\hline \multirow{3}{*}{$\begin{array}{c}\text { Intensidade } \\
\text { Sonora }\end{array}$} & Fraca & 0,7 & 0,75 & 0,3 & 0,3 \\
\cline { 2 - 6 } & Média & 0,1 & 0,15 & 0,29 & 0,5 \\
\cline { 2 - 6 } & Elevada & 0,2 & 0,1 & 0,5 & 0,2 \\
\hline \multirow{3}{*}{$\begin{array}{c}\text { Iluminação } \\
\end{array}$} & Claro & 0,55 & 0,45 & 0,55 & 0,4 \\
\cline { 2 - 6 } & Médio & 0,2 & 0,1 & 0,05 & 0,15 \\
\cline { 2 - 6 } & Escuro & 0,25 & 0,45 & 0,4 & 0,45 \\
\hline \multirow{2}{*}{ Espaço } & Amplo & 0,85 & 0,93 & 0,1 & 0,2 \\
\cline { 2 - 6 } & Apertado & 0,15 & 0,07 & 0,9 & 0,8 \\
\hline
\end{tabular}

trada (evidências) e uma variável de saída, ou seja, a hipótese Local. A Figura 8 ilustra a Rede Bayesiana, bem como as probabilidades a priori de todas as variáveis ${ }^{7}$. Por sua vez, as probabilidades condicionais das variáveis de evidência dada a hipótese Local utilizadas nas modelagem, são especificadas na Tabela 1. No modelo proposto, estas probabilidades são informações definidas pelo conhecimento de especialistas ou obtidas a partir de dados. Ressalta-se que o cenário do Robô Limpador é de cunho ilustrativo, de modo que não utiliza informações reais.

\footnotetext{
${ }^{7}$ Figura 8 foi criada por meio do software Netica, que implementa Redes Bayesianas sem inferência Fuzzy-Bayesiana. O software Netica está disponível em: https://www.norsys.com/netica.html.
} 


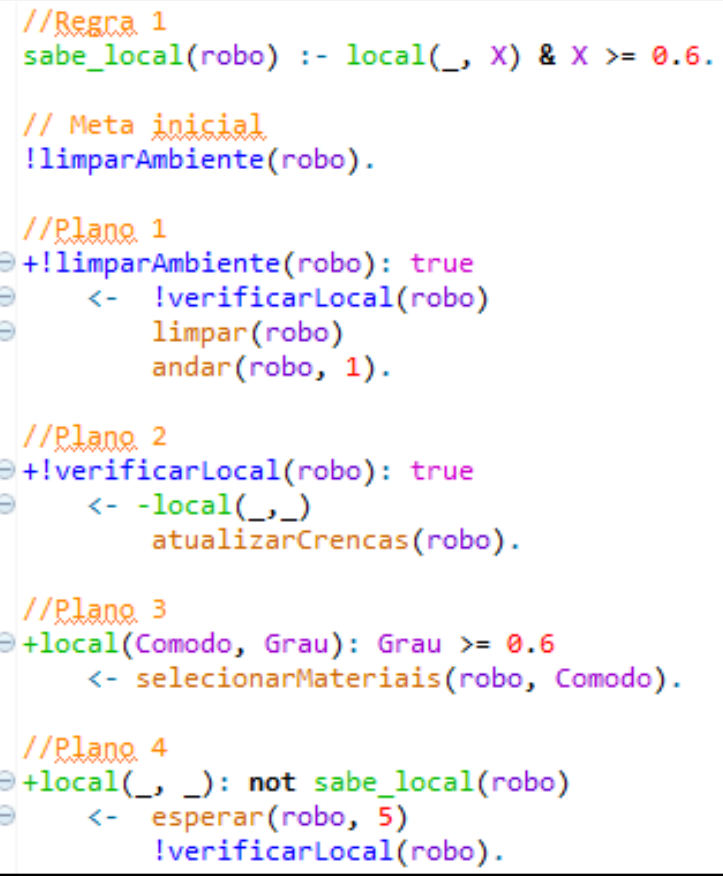

Figura 7. Base de Crenças e Planos do Robô Limpador

A modelagem das variáveis de evidência por meio de Conjuntos Fuzzy é ilustrada na Figura $9^{8}$. As variáveis referentes à intensidade sonora e à iluminação (Figura 9(a) e 9(c), respectivamente) possuem classes de pertinência não-dicotômicas, com sobreposição de estados. Em contrapartida, a variável referente ao espaço, foi modelada com classes dicotômicas (Figura 9(b)).

A seguir, serão descritas algumas simulações para visualizar o comportamento da distribuição de probabilidade ajustada pela imprecisão $\widetilde{P}\left(H_{h} \mid \mathscr{O}\right)$ (ver Equação (2)), conforme ocorre variação em alguma observação.

Quando uma observação é estimada em classes fuzzy dicotômica, como é o caso da observação referente ao espaço, existem apenas duas classes fuzzy cujos graus de pertinência geralmente são complementares. A Figura 10 apresenta o gráfico da distribuição da probabilidade para $\widetilde{P}\left(H_{h} \mid \mathscr{O}\right)$, com diferentes observações da variável Espaço. No gráfico, as observações são representadas por de tuplas (Medição, $\mu_{A m p l o}$, $\left.\mu_{\text {Apertado }}\right)$, cujos valores representam, respectivamente, a medição sensorial e os graus de pertinência da medição às classes fuzzy Amplo e Apertado.

Por meio das simulações ilustradas na Figura 10, é possível notar que conforme o grau de pertinência às classes Amplo e Apertado se aproxima de 0,5 (ou seja, conforme maior a imprecisão da medição), as probabilidades inferidas se aproximam das probabilidades a priori da variável de hipótese. Em contrapartida, quando o grau de pertinência é 1 para alguma classe fuzzy, a probabilidade estimada pela Inferência

\footnotetext{
${ }^{8}$ A modelagem de classes fuzzy e a estimação de graus de pertinência foram realizadas através da biblioteca jFuzzyLogic, disponível em http://jfuzzylogic.sourceforge.net/html/index.html.
}

Fuzzy-Bayesiana é idêntica a probabilidade estimada por uma Rede Bayesiana padrão, no caso da classe em questão ser confirmada como evidência.

A Figura 11 apresenta o gráfico da distribuição da probabilidade da hipótese ajustada sobre a imprecisão da variável não-dicotômica de Iluminação. No gráfico, as observações são representadas por de tuplas $\left(\right.$ Medição, $\left.\mu_{\text {Claro }}, \mu_{\text {Médio }}, \mu_{\text {Escuro }}\right)$, cujos valores representam, respectivamente, a medição sensorial da iluminação e os graus de pertinência da medição às classes fuzzy Claro, Médio e Apertado.

Por fim, na Figura 11 é apresentado o gráfico da distribuição da probabilidade da hipótese ajustada sobre a imprecisão da variável não-dicotômica de Intensidade Sonora. No gráfico, as observações são representadas por de tuplas (Medição, $\left.\mu_{\text {Fraca }}, \mu_{\text {Média }}, \mu_{\text {Elevada }}\right)$, cujos valores representam, respectivamente, a medição sensorial da intensidade sonora e os graus de pertinência da medição às classes fuzzy Fraca,Média e Elevada.

Utilizando variáveis não-dicotômicas como evidências (Figuras 11 e 12), nas simulações não ocorreram situações nas quais a probabilidade estimada se igualasse a probabilidade $a$ priori da hipótese. Entretanto, permanece a característica da Inferência Fuzzy-Bayesiana estimar a mesma probabilidade que uma Rede Bayesiana padrão, quando o grau de pertinência é 1 para alguma das classe fuzzy.

\section{Considerações Finais}

Com o intuito de obter a consciência situacional de um agente em nível de Percepção, este trabalho apresentou um modelo de fusão de dados. Para tratar a incerteza inerente de dados sensoriais, o modelo emprega inferência Fuzzy-Bayesiana. A saída do modelo é um conjunto de crenças com grau de certeza e são propagadas para os níveis de consciência situacional de Compreensão e Projeção, de modo a possibilitar o posterior raciocínio, planejamento e a tomada de decisão do agente. $\mathrm{O}$ uso da inferência Bayesiana permite estimar a probabilidade mesmo no caso de não serem observadas todas as evidências do ambiente, assim, se um sensor falhar, as crenças do agente ainda serão formadas.

Contudo, a abordagem Fuzzy-Bayesiana não permite a geração de crenças de saída difusas, pois o não é possível usar o modelo como um regressor. Esta característica, afeta agentes que precisem fazer uso da crença difusa em um controlador. Além disso, a crença difusa poderia ser empregada como entrada para outras redes Fuzzy-Bayesianas ou em sistemas fuzzy. Faz-se necessário ainda, considerar que o ambiente observado pelo agente possui características variantes no tempo. Esta variação pode ser introduzida no modelo através da incorporação de medições passadas à observação presente. Neste sentido, como trabalhos futuros, pretende-se explorar o uso de inferência de Redes Bayesianas Dinâmicas no modelo proposto.

Para exemplificar a necessidade de explorar a continuidade nos dados, será retomado o exemplo do Robô Limpador. 


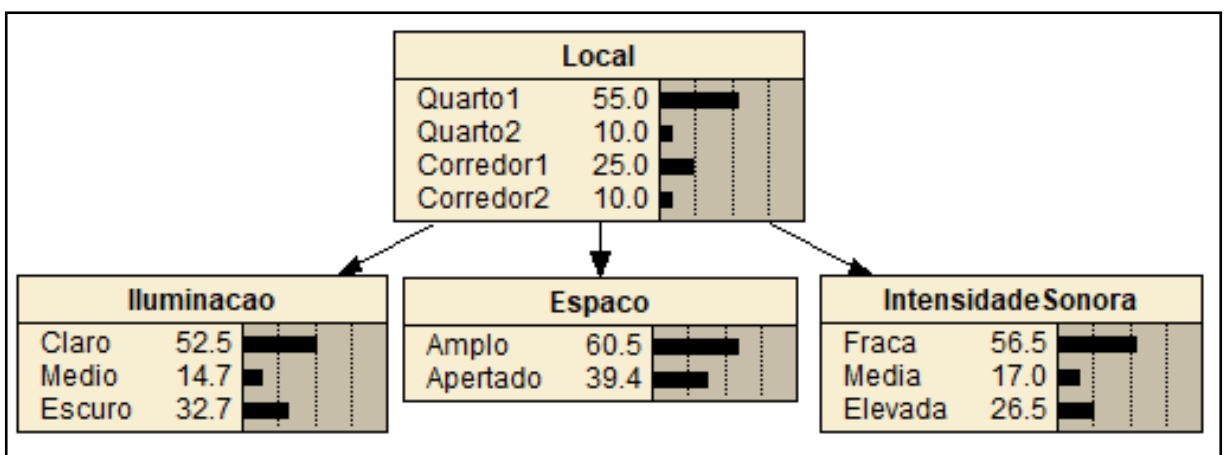

Figura 8. Rede Bayesiana para o Robô Limpador com as probabilidades a priori das variáveis

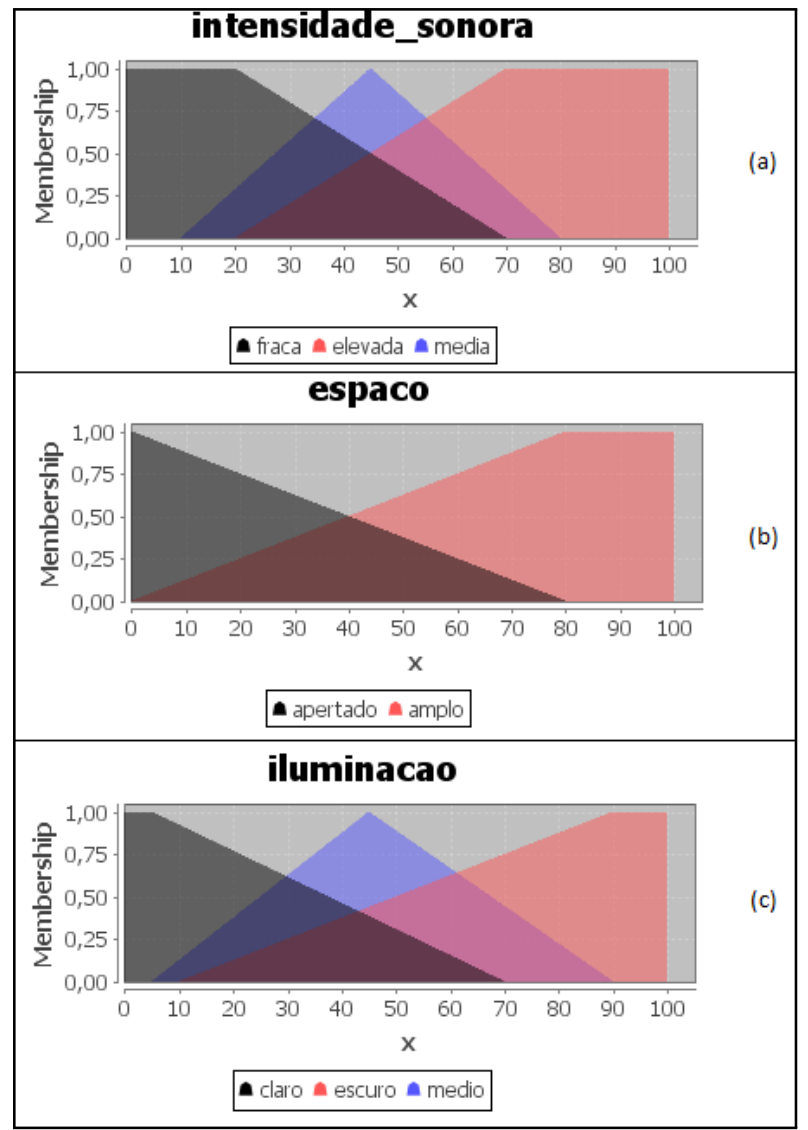

Figura 9. Modelagem Fuzzy das Variáveis de Entrada

Suponha que o robô estime sua localização a cada $k$ segundos e consiga apenas locomover-se um passo durante este intervalo. Considerando que as características de ambos os corredores $C_{1}$ e $C_{2}$ são similares, poderia ocorrer de o robô deduzir que sua localização em um instante $t$ seja $C_{1}$ e no instante $t+1$, seja $C_{2}$. Devido à disposição dos cômodos no cenário (Figura 2), sabe-se que não é possível movimentar-se de $C_{1}$ para $C_{2}$ em apenas um passo, assim, a dedução do robô seria inverossímil. Utilizando observações probabilísticas contínuas, o robô teria informações para julgar que, ao estar em $C_{1}$ no instante $t$, a probabilidade de continuar em $C_{1}$ ou mesmo de entrar em um dos quartos $Q_{1}$ ou $Q_{2}$ no instante $t+1$ é maior do que a probabilidade de ter atingido o corredor $C_{2}$.

Como trabalhos futuros, pretende-se implementar o modelo em um cenário robótico real, no qual seja possível explorar a tomada de decisão do agente e a escalabilidade da abordagem.

\section{Agradecimentos}

Os autores agradecem o apoio financeiro fornecido pela Coordenação de Aperfeiçoamento Pessoal de Nível Superior (CAPES).

\section{Contribuição dos Autores}

Este trabalho é um produto da dissertação de mestrado de Munyque Mittelmann, sob orientação de Aldo von Wangenheim e coorientação de Jerusa Marchi.

- Munyque Mittelmann - Pesquisador que desenvolveu o modelo proposto, implementou a proposta e escreveu o artigo.

- Jerusa Marchi: Coorientadora que auxiliou no processo de escrita, no desenvolvimento do modelo e também realizou uma revisão do artigo.

- Aldo von Wangenheim - Orientador que realizou uma revisão mais geral.

\section{Referências}

[1] WICKENS, C. D.; HOLLANDS, J. G. Engineering Psychology and Human Performance. 3. ed. Upper Saddle River, New Jersey: Prentice Hall, 2000. N/A. (N/A, N/A).

[2] ENDSLEY, M. R. Toward a theory of situation awareness in dynamic systems. Hum. Factors: J. Hum. Factors Ergon. Soc., v. 37, n. 1, p. 32-64, 1995.

[3] KOKAR, M. M.; MATHEUS, C. J.; BACLAWSKI, K. Ontology-based situation awareness. Inf. Fusion, v. 10, n. 1, p. 83-98, 2009. 


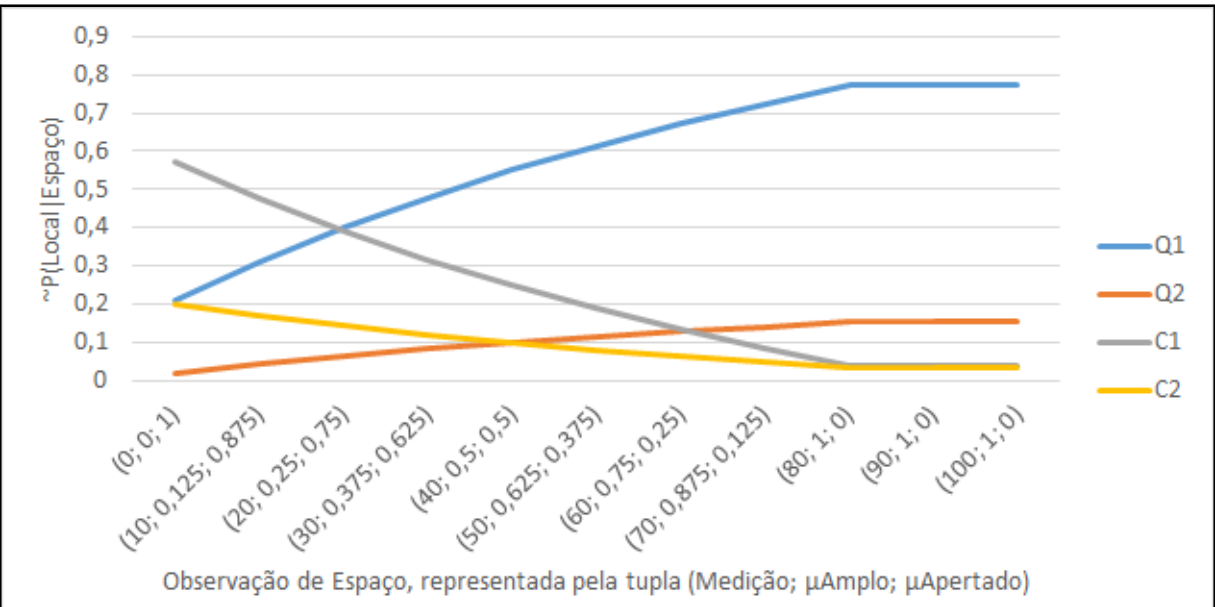

Figura 10. Distribuição da probabilidade da hipótese ajustada pela imprecisão da evidência Espaço

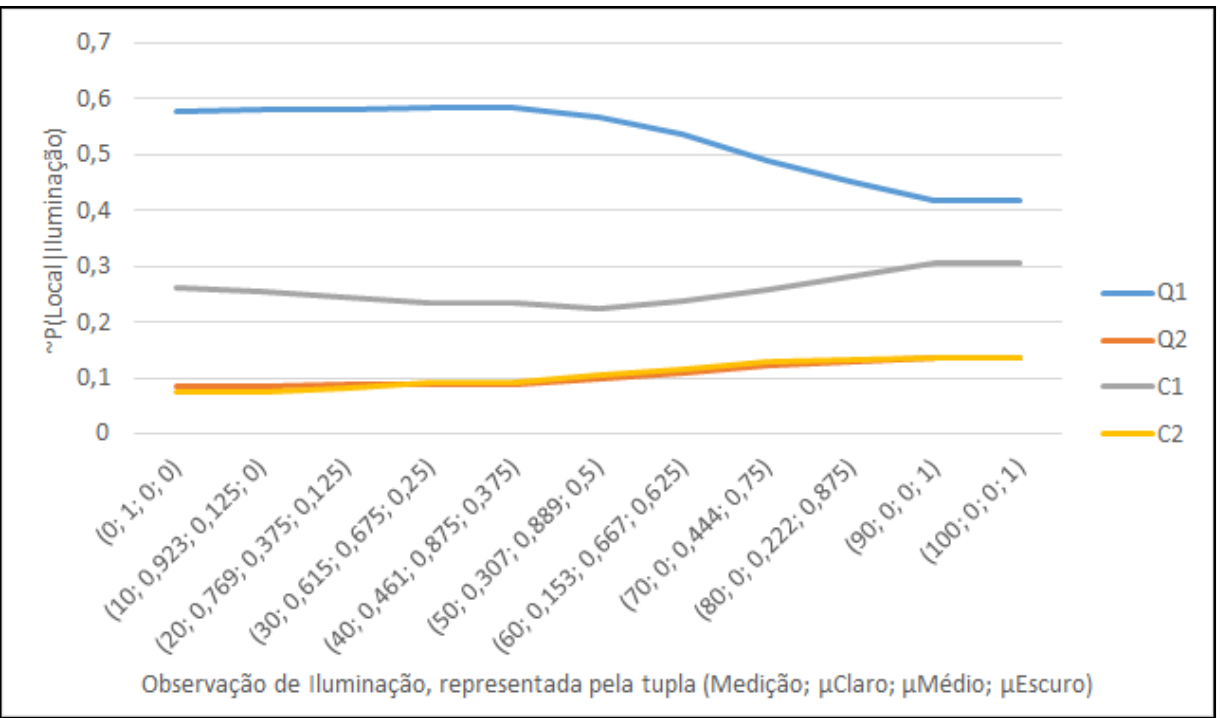

Figura 11. Distribuição da probabilidade da hipótese ajustada pela imprecisão da evidência Iluminação

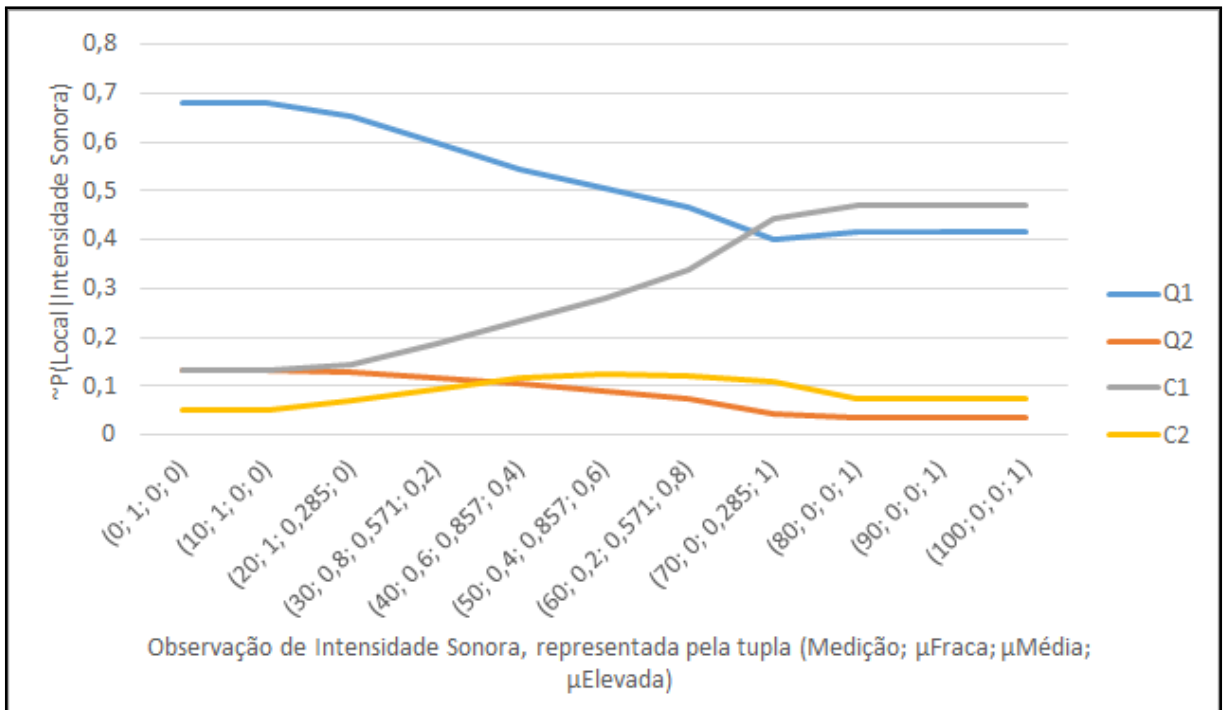

Figura 12. Distribuição da probabilidade da hipótese ajustada pela imprecisão da evidência Intensidade Sonora 
[4] CHEN, L.; NUGENT, C.; AL-BASHRAWI, A.

Semantic data management for situation-aware assistance in ambient assisted living. In: . New York, NY, USA: ACM, 2009. (iiWAS '09, N/A), p. 298-305.

[5] GOLESTAN, K. et al. Situation awareness within the context of connected cars: A comprehensive review and recent trends. Inf. Fusion, v. 29, n. N/A, p. 68-83, 52016.

[6] INSAURRALDE, C. C.; PETILLOT, Y. R. Capabilityoriented robot architecture for maritime autonomy. Robot. Auton. Syst., v. 67, n. N/A, p. 87-104, 52015.

[7] STEINBERG, A. N.; BOWMAN, C. L. Rethinking the jdl data fusion levels. NSSDF Conf. Proc., N/A, n. c, p. 1-18, 2004.

[8] WHITE, F. E. A model for data fusion. In: . N/A: N/A, 1988. (N/A, v. 2), p. 149-158.

[9] KHALEGHI, B. et al. Multisensor data fusion: A review of the state-of-the-art. Inf. Fusion, v. 14, n. 1, p. 28-44, 2013.

[10] LI, Y.; CHEN, J.; FENG, L. Dealing with uncertainty: A survey of theories and practices. IEEE Trans. Knowl. Data Eng., v. 25, n. 11, p. 2463-2482, 2013.

[11] LI, M.; FENG, X. Situation assessment based on bayesian networks. In: . N/A: N/A, 2008. (N/A, N/A), p. 1-3.

[12] ZHI-FU, S.; HAI-YAN, L. Intelligent Situation Fusion Assessment Using Bayesian Networks. In: 2009 Second International Conference on Information and Computing Science. [S.1.: s.n.], 2009. v. 4, p. 212-215.

[13] DAS, S.; GREY, R.; GONSALVES, P. Situation assessment via Bayesian belief networks. Proceedings of the Fifth International Conference on Information Fusion. FUSION 2002. (IEEE Cat.No.02EX5997), v. 1, p. 664-671, 2002. Disponível em: 〈http://ieeexplore.ieee.org/lpdocs/ epic03/wrapper.htm?arnumber=1021218 .

[14] LI, X.; XIAO, L. The application of situation assessment based on fuzzy algorithm in emergency management. In: . N/A: N/A, 2010. (N/A, N/A), p. 295-297.

[15] PRABHU, A. et al. Decision fusion using type-1 fuzzy logic for situation assessment in aviation scenarios. In: 2014 International Conference on Advances in Electronics Computers and Communications. [S.1.: s.n.], 2014. p. 1-6.

[16] LIANG, Y. An Approximate Reasoning Model for Situation and Threat Assessment. In: Fourth International Conference on Fuzzy Systems and Knowledge Discovery (FSKD 2007). [S.1.: s.n.], 2007. v. 4, p. 246-250.

[17] CHAI, H.; WANG, B. A hierarchical situation assessment model based on fuzzy bayesian network. In: International Conference on Artificial Intelligence and Computational Intelligence. N/a. N/A: Springer, 2011, (N/A, N/A). p. 444-454.

[18] NADERPOUR, M.; LU, J.; ZHANG, G. A fuzzy dynamic bayesian network-based situation assessment approach. In: 2013 IEEE International Conference on Fuzzy Systems (FUZZ-IEEE). [S.1.: s.n.], 2013. p. 1-8.
[19] NADERPOUR, M.; LU, J.; ZHANG, G. The explosion at institute: Modeling and analyzing the situation awareness factor. Accident Analysis and Prevention, Elsevier Ltd, v. 73, p. 209-224, 2014. Disponível em: 〈http://dx.doi.org/10.1016/j.aap.2014.09.008〉.

[20] NADERPOUR, M.; LU, J.; ZHANG, G. An intelligent situation awareness support system for safety-critical environments. Decision Support Systems, Elsevier B.V., v. 59, n. 1, p. 325-340, 2014. Disponível em: $\langle$ http://dx.doi.org/10.1016/j.dss.2014.01.004〉.

[21] TOMASO, E. D.; BALDWIN, J. F. An approach to hybrid probabilistic models. International Journal of Approximate Reasoning, v. 47, n. 2, p. 202-218, 22008. Disponível em: 〈https://www.sciencedirect.com/science/ article/pii/S0888613X07000485 $\rangle$.

[22] WANG, Y. F. et al. Fire probability prediction of offshore platform based on Dynamic Bayesian Network. Ocean Engineering, Elsevier Ltd, v. 145, n. April, p. 112-123, 2017. Disponível em: 〈https: //doi.org/10.1016/j.oceaneng.2017.08.035〉.

[23] BRIGNOLI, J. T. Um Modelo para Suporte ao Raciocínio Diagnóstico Diante da Dinâmica do Conhecimento sobre Incertezas. Tese (Doutorado), N/A, 2013.

[24] BRIGNOLI, J. T. et al. A fuzzy-bayesian model based on the superposition of states applied to the clinical reasoning support. IntelliSys 2015 - Proc. 2015 SAI Intell. Syst. Conf., N/A, n. N/A, p. 210-219, 2015.

[25] KOLLER, D.; FRIEDMAN, N. Probabilistic graphical models: principles and techniques. N/a. N/A: MIT press, 2009. N/A. (N/A, N/A).

[26] ZADEH, L. Fuzzy sets. Inf. Control, v. 8, n. 3, p. 338-353, 1965.

[27] VIERTL, R. Is it necessary to develop a fuzzy bayesian inference? In: Probability and Bayesian Statistics. N/a. Boston, MA: Springer US, 1987, (N/A, N/A). p. 471-475.

[28] VIERTL, R. Fuzzy bayesian inference. In: Soft methods for handling variability and imprecision. N/a. Berlin, Heidelberg: Springer, 2008, (N/A, N/A). p. 10-15.

[29] VIERTL, R. Modeling of fuzzy measurements in reliability estimation. In: Reliability Data Collection and Use in Risk and Availability Assessment. N/a. Berlin, Heidelberg: Springer, 1989, (N/A, N/A). p. 206-211.

[30] VIERTL, R.; HULE, H. On bayes ' theorem for fuzzy data. Stat. Pap., v. 32, n. N/A, p. 115-122, 1991.

[31] VIERTL, R. Statistics with fuzzy data. In: RICCIA, G. D.; KRUSE, R.; VIERTL, R. (Ed.). Vienna: Springer Vienna, 1995. (N/A, N/A), p. 33-49.

[32] PADGHAM, L.; WINIKOFF, M. Developing intelligent agent systems: A practical guide. N/a. N/A: John Wiley

Sons, 2005. v. 13. (N/A, v. 13). 